marked suppression and divergence. Secondly, as there is no need to increase power of abduction; it is unnecessary to insist on S.M.P., though this is, of course, an advantage. The power of convergence may be greatly developed by practice on a pencil, etc., as for convergence deficiency, and one often finds that S.M.P. is attained readily after such a stimulus to normal binocular vision.

Should the abnormal correspondence not yield readily to treatment after operation, the angle being satisfactory, it is probably wisest to leave the case alone, with observation at intervals, so that when time has done its bit in breaking down the abnormal correspondence, one may jump in and deal the death blow.

Although the treatment as here indicated includes some timehonoured methods, the omission of several useful tricks will be obvious to orthoptists. My aim has been to make a statement as simple as possible.

\title{
CONTACT LENS IN CASES OF NEUROPARALYTIC KERATITIS
}

\author{
BY - \\ M. KLEIN \\ , LONDON
}

AMONG the indications for the use of contact lenses neuroparalytic keratitis does not seem to have attracted much attention. This record of their use in two cases may therefore be of interest.

1. Miss V. P., aged 39 years. Had several operations for right sided acoustic tumour. The last operation left her with a complete right facial palsy and trigeminal anaesthesia. Neuroparalytic keratitis of the right eye developed, which eventually made tarsorraphy necessary, but in spite of this, the state of the cornea did not improve. After a year, though the lids were still sutured, the condition of the eye seemed hopeless. The therapeutic effect of a contact lens was then tried as a last resort (August, 1942). Rapid improvement followed, and withih 2-3 weeks the eye became white, the cornea cleared up considerably, except at the central area where a dense scar remained. The patient is wearing the contact lens regularly from 7 a.m. to 1 p.m. when it is removed for about halfan-hour, and generally worn again till about 9 p.m.

An incident emphasised the value of the contact lens. In November, 1942, the patient accidentally broke the contact lens. The eye became inflamed within a few hours, and by the following 
day the corneal infiltrate was extensive. The development of such a severe keratitis in such a short time was astonishing. The patient was then supplied with a new contact lens, and within a week the eye quietened down again.

2. Mrs. A.D., aged 58 years. Five weeks after alcohol.injection for right trigeminal neuralgia, neuroparalytic keratitis developed. When first seen a fortnight after the onset of the affection the area involved extended centrally for about $7 \mathrm{~mm}$. diameter. The patient was supplied with a contact lens, which she is wearing for about 12 hours a day, with a break of about half to one hour at mid-day. The patient has now been wearing the contact lens for four months. The cornea has cleared considerably, though there is a central nebula. Vision without contact glass but with correction is $6 / 24$, and with contact glass $6 / 12$.

\section{Comment :}

1. Fitting.-From the fitting point of view the anaesthesia of the eye is a slight initial advantage. The taking of the impression can be made without the use of any anaesthetic. When the contact lens is put in, the reaction of the eye makes it easy to tell whether the fitting of the contact lens is satisfactory. A wrong fitting causes irritation (hyperaemia, discharge, etc.). In the absence of subjective signs in the neuroparalytic cornea the reaction of the eye shows readily any deficiency of the fitting.

2. Indications. - It appears that the use of contact lenses is warranted as an alternative to tarsorraphy. It gives better protection for the eye as was shown in the first case. From the aesthetic point of view it has distinct advantages. In the first case the patient was much happier with the contact lens which has enabled her to discard the shade she previously wore to hide the deformity caused by the sutured lids. Moreover, there need be no reluctance in trying a contact lens when there is hesitation about undertaking such a disfiguring procedure as tarsorraphy.

My thanks are due to Miss J. M. Fleming for referring the first case to me, and Miss A. Logan Adam for the second case.

\section{ORBITAL EMPHYSEMA SIMULATING CELLULITIS BY \\ C. L. Conor O'MALley \\ GALWAY}

ORBITAL emphysema is apparently a rare condition as indicated by the literature at my disposal on the subject. The majority of cases are described as following a benign course, not necessitating surgical 